\title{
Die Conceptual-Integration-Forschung als Anregung für die komparatistische Selbstreflexion?
}

\author{
Antje Wischmann
}

Online publiziert: 17. Juli 2019

(C) Der/die Autor(en) 2019

Zusammenfassung Dieser Beitrag geht von der Definition der Metapher als einem verkürzten Vergleich aus und untersucht zwei exemplarische Fälle von Metaphern/ Vergleichen aus der schwedischen und der norwegischen Literatur. Dabei werden neuere Ansätze von Line Brandt adaptiert, die sich conceptual integration im Umfeld sowohl der kognitiven Semiotik als auch der Literaturwissenschaft widmet. Dabei wird die Interaktion von mindestens zwei semantischen Domänen in einem blend untersucht. Obwohl ein solcher mehrschrittiger Kombinationsvorgang oft in ein unverwechselbares Resultat mündet, lassen sich auch allgemein übertragbare Erkenntnisse aus diesen bedeutungserzeugenden Prozessen gewinnen. Die Korrespondenzbeziehungen zwischen den input spaces tragen so zur Beleuchtung komparativer Verfahrensweisen bei: Alle Teilschritte sind pfadabhängig und bahnen irreversible Deutungsprozesse an, die sich retrospektiv nur mehr schwer nachvollziehen lassen.

Schlüsselwörter Metapher · Vergleich · Blending · Conceptual Integration · Komparatistik · Skandinavische Literatur

\section{Research in Conceptual-Integration as a Stimulus for Self-Reflection in Comparative Literary Studies?}

\begin{abstract}
This article investigates two exemplary literary cases of metaphors/ comparisons from Swedish and Norwegian literature, viewing metaphors as abbreviated comparisons and analysing them with the help of the conceptual integration theory developed by Line Brandt, an exponent of cognitive semiotics. A blend as a result of conceptual integration is generated by the processes of exchange between
\end{abstract}

\footnotetext{
A. Wischmann $(\bowtie)$

Institut für Europäische Vergleichende Sprach- und Literaturwissenschaft EVSL, Abteilung

Skandinavistik, Universität Wien, Wien, Österreich

E-Mail: antje.wischmann@univie.ac.at
} 
at least two interacting semantic domains. This is an emergent unicum but also a conceptual entity and it is fruitful to scrutinize its mechanisms of construction. The correspondences that are established between the input spaces justify the application of the integration process to the method of comparison: It may be beneficial to break down and comprehend in detail the various steps involved in interpretation. This process explains why it is difficult to re-establish an unbiased perspective after carrying out a comparison: our ideas about the input spaces are enduringly recast during the process of comparing.

Keywords Metaphor - Blending · Conceptual Integration · Comparative Literary Studies $\cdot$ Scandinavian Studies

\section{Dem Vergleichen auf der Spur}

\subsection{Bildhaftes Kommunizieren}

Metaphern und Vergleiche sind gleichermaßen faszinierend und regen immer wieder dazu an, die bildlichen Konzeptualisierungen und die immanenten semantischen Austauschbeziehungen nachzuvollziehen. ${ }^{1}$ In jüngster Zeit wird die mögliche allgemeine Erkenntnis, die sich aus vergleichenden Tätigkeiten und wissenschaftlichen Operationen des Vergleichs ableiten könnte, in den Blick genommen, was nicht zuletzt dem neuen Forschungsprofil >Literatur und Wissen $<\mathrm{zu}$ verdanken ist. In der Einleitung zu ihrer Anthologie Die Welt beobachten (2015) über vergleichende Praktiken in kulturwissenschaftlichen und historischen Disziplinen kreisen Angela Epple und Walter Erhart diese Möglichkeiten der Erkenntnisgewinnung wie folgt ein:

»Die Bedeutung der Praxis und des Kontexts für den Akt des Vergleichens lässt sich bereits an einer seiner kleinsten sprachlich-rhetorischen Formen beobachten, der Metapher, einem Vergleich, bei dem der Vergleichspartikel >wie $<$ fehlt, der gerade deshalb aber die impliziten Grundlagen und Funktionen eines solchen Vorgangs verdeutlicht. Die alltägliche rhetorische Praxis des Metaphorisierens ist sowohl kognitiv-sprachlich als auch kommunikativ-kulturell situiert, sie erzeugt [...] eine vom tertium comparationis angestoßene Bewegung von Gemeinsamkeiten und Unterschieden, in der die comparata immer auch verändert werden, in der neue Kontexte hervorgebracht und neue mentale und kulturell wirksame Wissensbestände produziert werden. $\ll^{2}$

Der vorliegende Beitrag beachtet die im Zitat genannte kognitiv-sprachliche und kommunikativ-kulturelle Einbettung des Vergleichens und diskutiert einen Zugang

\footnotetext{
1 Für zahlreiche wertvolle Hinweise danke ich Annika Krummacher.

2 Epple, Angelika/Erhart, Walter: »Die Welt beobachten - Praktiken des Vergleichens«. In: Dies. (Hg.): Die Welt beobachten. Praktiken des Vergleichens. Frankfurt a.M./New York, 2015, S. 7-31, S. 20. Zum Verständnis der Metapher als > Vergleich ohne wie< siehe Brandt, Line: »Metaphor and the Communicative Mind «. In: Journal of Cognitive Semiotics, Vol. 5, No. 1-2 (2011), S. 37-72, S. 60; Kövecses, Zoltan: Metaphor. Oxford/New York 2010, S. 267; Skirl, Helge/Schwarz-Friesel, Monika: Metapher. Heidelberg 2007, S. 11.
} 
aus der aktuellen Forschung zur kognitiven Semiotik, indem ein Teilgebiet der >conceptual integration theory< adaptiert und für die analytische Erschließung von literarischen Metaphern und Vergleichen erprobt wird. Metaphern und Vergleiche erzeugen selbst konzeptuelle Integrationen und sind zugleich Resultate integrativer Prozesse zwischen Bedeutungsdomänen. Dieser Beitrag behandelt vornehmlich literarisch-ästhetische Vergleiche, um abschließend vergleichende Praktiken in der Komparatistik einzubeziehen, die von einer literaturwissenschaftlich-linguistischen Betrachtung sicherlich profitieren könnte. Indem nämlich Vergleiche ein konzeptuell und sprachlich selbstbezügliches Potenzial offenbaren, thematisieren oder hinterfragen sie ihre eigenen Vergleichsparameter und Vorannahmen.

Die Forscherin Line Brandt propagiert, dass jeweils eine systematische semantische und eine literarische Interpretation ineinandergreifen sollten - ihre umfassende Monographie The Communicative Mind aus dem Jahr $2013^{3}$ liefert neben einem detaillierten Forschungsabriss zur kognitiven Semiotik eigene modellhafte Zugänge, wobei das Kapitel »Conceptual Integration in Semiotic Meaning Construction « für meinen Beitrag eine Schlüsselfunktion übernimmt. Der Terminus >conceptual integration< löst den Begriff des >blending< ab, der seine populäre Hochphase bereits überschritten hat. Die Annahme von semantischen Interferenzen in George Lakoffs und Mark Johnsons Modellierung der »metaphorical mappings « und »cross-domain correlations $\aleph^{4}$ bildet seit 1980 den Ausgangspunkt für unterschiedlich gewichtete Theoriebildungen.

The Communicative Mind synthetisiert Brandts eigene Forschungsarbeiten des letzten Jahrzehnts sowie die ihrer akademischen Lehrenden Gilles Fauconnier, Mark Turner, Eve Sweetser, Ron Langacker und nicht zuletzt die Publikationen ihres Vaters Per Aage Brandt, der in den 1990er Jahren Initiator des Aarhusianer Zentrums für Semiotik (in Dänemark) war. Für die Metaphernforschung haben sich die von L. und P. Brandt gemeinsam verfassten Artikel »Making sense of a blend « sowie »Cognitive Poetics and Imagery « (beide 2005) ${ }^{5}$ mittlerweile als grundlegend erwiesen.

In Line Brandts Arbeiten ist eine wachsende Distanzierung von Turners Pionierwerk The Literary Mind $(1996)^{6}$ zu erkennen, auf das der Titel The Communicative Mind natürlich anspielt: Turners Versuch, mit der >blending theory< die Vermischung von Kategorien, die sogenannte Mehrfachperspektivierung oder die Überblendung

\footnotetext{
${ }^{3}$ Brandt, Line: The Communicative Mind. A Linguistic Exploration of Conceptual Integration and Meaning Construction. Newcastle-upon-Tyne 2013. Im Folgenden abgekürzt als Brandt 2013.

${ }^{4}$ Lakoff, George/Johnson, Mark: »Afterword, 2003«. In: Dies.: Metaphors we live by. With a new afterword. Chicago 2003/1980, S. 243-276, S. 246 u. 245. Zur stichhaltigen Kritik an Lakoff u. Johnson siehe Eder, Thomas: »Zur kognitiven Theorie der Metapher. Eine kritische Bestandsaufnahme«. In: Franz Josef Czernin/Thomas Eder (Hg.): Zur Metapher. Die Metapher in Philosophie, Wissenschaft und Literatur. München 2007, S. 167-195. (Sämtliche neuronalen Aspekte der Contemporary Metaphor Theory CTM sind mittlerweile veraltet, man erinnere sich nur an das Debakel um die sogenannten Spiegelneuronen.).

5 Brandt, Line/Brandt, Per Aage: »Making sense of a blend. A cognitive-semiotic approach to metaphor«. In: Annual Review of Cognitive Linguistics, Vol. 3 (2005), S. 216-249 und Dies.: »Cognitive Poetics and Imagery«. In: European Journal of English Studies, Vol. 9, No. 2 (2005), S. 117-130.

6 Turners Buch wurde 2000 ins Dänische übersetzt, um die Vermittlung an Studierende zu erleichtern, und mit einem Vorwort von P. Brandt versehen - dies hebt den Grad der Popularität von The Literary Mind umso deutlicher hervor (Turner, Mark: Den littercere bevidsthed. En kognitiv teori om tankens og sprogets oprindelse. übs. v. Kristian Ditlev Jensen und Tom Havemann, Kopenhagen 2000).
} 
von Bedeutungsdomänen zu erklären, stellt sich 2005 und erst recht in den kritischen Auslassungen der Anthologie Kognitiv Poetik (2009) 7 aus Sicht von L. und P. Brandt als zu breit gefasst und universalistisch, wenn auch weiterhin inspirierend dar. Wichtige Anliegen von The Communicative Mind sind demnach sowohl Relativierung und Feinabstimmung bisheriger Blending-Forschung als auch eine methodische Operationalisierbarkeit des Conceptual-Integration-Ansatzes für Linguistik und Literaturwissenschaft.

Auch wenn die Metaphernforschung den Ankerpunkt der kognitiven Semantik bildet, visiert mein Artikel keineswegs an, Gesetzmäßigkeiten von mentalen Repräsentationen oder Projektionen, wie denen der Imagination und der Fiktion, aufzudecken. ${ }^{8}$ Stattdessen konzentriere ich mich auf die von Brandt dargelegte Bedeutungsgenerierung von Metaphern ${ }^{9}$ sowie auf >mental space blends $<$, die eine Interaktion zwischen unterschiedlichen semantischen Domänen umfassen.

Im Folgenden werden nach der Einführung in das adaptierte Modell zwei Fallbeispiele erörtert: Elmer Diktonius' Gedicht »Södergran « wird herangezogen, um ausgewählte metaphorische Prozesse genau nachzuvollziehen: Dabei tritt zum einen die Pfadabhängigkeit einer solchen Bedeutungsgenese hervor, zum anderen drängt sich die strukturierende Kraft des >Wissens im Nachhinein` auf, so dass rückschauend eine schrittweise Untersuchung re- und neu-konstruiert werden muss.

Während der Gedichttext eine abgegrenzte Lektüreeinheit bildet, wird für das zweite Fallbeispiel ein Dialogabschnitt aus einem Ibsen-Drama gewählt, also eine Lexie aus einem Werk herausgelöst. ${ }^{10}$ In der Gesprächsszene werden Metaphern und Vergleiche auf markante Weise verhandelt, weil sich die Diskussion der Figuren als Stellvertreterkonflikt erweist und eine metapoetische Dimension hervortritt. Die Dramenszene nehme ich zum Anlass, um weitere typologische Varianten von >mental space blends $<$ im Hinblick auf die theatrale Inszenierung zu skizzieren.

\subsection{Introspektion und kommunikative Kontextualisierung}

Aus der strukturalistischen Vorgeschichte semiotischer Ansätze scheint sich eine starke Fokussierung auf den Textsinn zu ergeben, die L. Brandts The Communicative Mind indessen durch eine Betonung der Kommunikationssituation und des konkreten Sprachgebrauchs überwindet: Die Kontextualisierung erweitert die Introspektion auf geradezu befreiende Weise. ${ }^{11}$ So finden performative Umsetzungen, die körperliche Situierung sowie mediale Transformationen Berücksichtigung oder sind

\footnotetext{
7 Brandt, Line/Kjørup, Frank: »Introduktion«. In: Dies. (Hg.): Kognitiv Poetik. Aarhus 2009, S. 7-42 sowie in den Kommentaren von Per Aage Brandt in den Fußnoten, passim.

8 Vgl. die Kritik an Lakoffs u. Johnsons Prämissen der Metaphernbetrachtung bei Eder: »Zur kognitiven Theorie der Metapher«, S. 188-195. Eders Würdigung der Conceptual-Integration-Forschung (vgl. ebd., S. 194) verstehe ich als wegweisende Aufforderung, zukünftig die Risiken des Reduktionismus zu vermeiden.

9 Vgl. Brandt 2013, S. 231-298.

10 Die Textkorpora festzulegen und damit zu entscheiden, welche Vergleichsparameter oder inter- und intratextuellen Verweisungen potenziell zum Tragen kommen könnten, bildet den ersten Arbeitsschritt auch von komparatistisch Forschenden, der selten ausreichend reflektiert wird.

11 Insofern ist L. Brandts Zugang über den Vorwurf erhaben, in einem altmodisch strukturalistischen Sinne $>$ langue< als eine Abstraktion zu untersuchen.
} 
zumindest anschlussfähig. ${ }^{12}$ Brandt stellt den Begriff der >énonciation< nach Émile Benveniste ${ }^{13}$ an den Anfang ihrer Überlegungen, um die Verankerung jeglicher sprachlicher Äußerung in einem situationsabhängigen, konkreten Kommunikationskontext hervorzuheben. >Énonciation< vergegenwärtigt die präsuppositionierte Faceto-face-Situation aller sprachlichen Aktivitäten. Die Sprecher handeln jeweils unter persönlichen Voraussetzungen, indem und während sie eine Äußerung an einen Adressaten richten, und sie tun dies, wie schon Benveniste betont, stets in einer bestimmten Absicht. Ihren Analyseansatz beschreibt Brandt als minutiöse und entschleunigte $\gg$ Offenlegung des konzeptuellen Interpretationsprozesses $«,{ }^{14}$ an dessen vorläufigem Abschluss die höchste Ebene der Bedeutungsgenerierung wieder auf die Prämisse zurückzubeziehen sei, ${ }^{15}$ um die >énonciation< vollgültig bestimmen zu können. Aus der kognitiven Semiotik und der Narratologie stammen die Termini >Schema < und >Szenario<: Der Begriff Schema bezeichnet eine Form, mit deren Hilfe stabile mentale Repräsentationen von räumlich-zeitlichen Prozessen generiert werden. Diese makrostrukturellen Formen werden nicht selten als Konzepte bezeichnet, wie z. B. BEWEGUNG. Der Begriff Szenario umfasst eine Ereignisabfolge, die sich aus einer gegebenen oder potenziellen Handlung herleitet. ${ }^{16}$ Zur Bestimmung des jeweiligen >Szenarios « werden laut Brandt folgende Einheiten identifiziert: »partial and temporary representional structures which speakers construct when thinking or talking $\ll{ }^{17}$ Es geht demnach um dynamische Vorstellungen und Konzeptualisierungen im >mental space<, die als ein Set aus strukturellen Einheiten evoziert werden. ${ }^{18}$

12 Die Kritik, die Vera Stadelmann noch 2012 an der angeblichen semiotischen Einseitigkeit und der Vernachlässigung der sozialen Interaktion in Brandts Forschungen übt, hat sich auf diese Weise weitgehend erledigt, da Line Brandt 2013 den Forschungsstand aktualisiert (vgl. Stadelmann, Vera: Language, Cognition, Interaction. Conceptual Blending as Discursive Practice. Gießen 2012, S. 42-72, In: http://geb.unigiessen.de/geb/volltexte/2012/8854/pdf/StadelmannVera_2012_04_25.pdf [9.2.2017]). Nichtsdestotrotz würdigt Stadelmann die pragmatische Erweiterung, die Hinwendung zur konkreten Kommunikationssituation und besonders nachdrücklich die in Brandts Dissertation ausgearbeitete Typologie der Integrationsarten (vgl. ebd. S. 48-49 u. S. 275-276; Bezug nehmend auf Brandt, Line: Language and enunciation. A cognitive inquiry with special focus on conceptual integration and semiotic meaning construction. Aarhus 2010, wo die Typologie erstmalig publiziert wurde).

13 Im Dänischen wird >énonciation « mit »udsigelse« (in Absetzung von »udsagn«) übersetzt, in etwa »Aussagehandlung .

14 Brandt 2013, S. 238.

15 Das Analysemodell entspricht der Konstellation von Repräsentamen, Objekt und Interpretant in der Peirceschen Semiotik, die hier zu >presentation space<, >reference space< und >blend < umgemünzt sind: Der Interpretant kann in einem weiteren Deutungsprozess wieder zu einem Repräsentamen werden und eine weitere Bedeutungsgenerierung einleiten. Genauso kann ein >blend < wieder zu einem neuen >semiotic base space $<$ werden, auf dem weitere Bedeutungsdomänen oder >mental spaces $<$ aufbauen könnten (vgl. Brandt 2013, S. 282-283).

16 Vgl. Brandt 2013, S. 19-20.

17 Brandt 2013, S. 246.

$18 \mathrm{Zu}$ statischen/dynamischen Schemata, Frames und Scripts als anschlussfähigen diskurslinguistischen Begriffen siehe Spitzmüller, Jürgen/Warnke, Ingo H.: Diskurslinguistik. Berlin/Boston 2011, S. 92-93. Brandts Begriffe Schema und Szenario leiten dazu an, Satzinhalte (eine oder mehrere Propositionen) zu erschließen. Dies entspricht aus diskurslinguistischer Sicht einer »propositionsorientierten intratextuellen Analyse (ebd., S. 153, siehe auch S. 165). 


\begin{tabular}{|l|l|}
$\begin{array}{l}\text { } \\
\text { meaning space } \\
\text { (Sonderfall: post-emergente Bedeutung) }\end{array}$ \\
$\begin{array}{l}4 \\
\text { blending space } \\
\text { (entstanden durch die Projektionen des presentation space und des reference space } \\
\text { infolge des Mappings) }\end{array}$ & input space \\
\hline 3 & M a p p i n g \\
input space & $\begin{array}{l}\text { reference space } \\
\text { hier enthalten: target } \\
\text { (Bildempfänger) mit einem } \\
\text { bestimmten Szenario }\end{array}$ \\
$\begin{array}{l}\text { presentation space } \\
\text { hier enthalten: source } \\
\text { (Bildspender) mit einem } \\
\text { bestimmten Szenario }\end{array}$ & \\
\hline $\begin{array}{l}2 \\
\text { semiotic base space }\end{array}$ & \\
\hline 1 & \\
ground: Phänowelt, discourse, Situation & \\
\hline
\end{tabular}

Abb. 1 Vereinfachtes Diagramm der >conceptual integration<

Für ihre graphische Darstellung von Prozessen der >conceptual integration < wählt L. Brandt ein Modell aus kreisförmigen $>$ spaces $<{ }^{19}$ zwischen denen sich allmählich feine Netzwerkbeziehungen bilden, wodurch die komplexe Gesamtstruktur nach unten und zu den Seiten >weiterwächst<. Brandts Analyse des vieldiskutierten Metaphernbeispiels » This surgeon is a butcher ${ }^{20}$ ist besonders eingängig, weil sie die Kommunikationsabsicht greifbar werden lässt, die erst aus dem Zusammenspiel der semantischen Domänen hervorgeht.

Um zu einer Adaption im Rahmen der komparatistischen Literaturwissenschaft zu ermutigen, wähle ich ein vereinfachtes Diagramm in Form einer Schichtung von unten nach oben. Diese Stufenfolge zeigt jedoch keine teleologische Entwicklung an, wie die Erläuterung der Fallbeispiele verdeutlichen wird (Abb. 1).

Auf der Ebene des Fundaments (1) findet die >énonciation< als situative Kommunikation statt - ein Akzent, der dem Modell auf attraktive Weise Konkretion verleiht. ${ }^{21}$ Nach der Bestimmung von Sprechern und Adressaten sowie einer ersten Einschätzung von >énonciation < und Aussage im Kontext des Gebrauchs (Ebene 2) wird die Repräsentation der >énonciation< von Seiten der Kommunizierenden (Ebene 3) untersucht. Der Gegenstand, über den Aussagen getroffen werden, in Brandts Beispiel ein Chirurg, gehört dem >reference space < an. Der Bildspender (bei Brandt ein Schlachter) geht in den >presentation space< ein. Richtet sich ein Sprecher mit der besagten kritischen Bemerkung über den Chirurgen an einen Adressaten, wird für beide Kommunizierende ein Prozess der Herstellung mentaler Bezüge (Mapping)

19 Vgl. Brandt 2013, S. 262, Figure 3-3.

20 Vgl. ebd., S. 237-298.

21 Vgl. ebd., S. 286 u. S. 296-297. 
eingeleitet, was ein Charakteristikum des Modells ausmacht: Bevor ein >blend< entsteht, muss sich ein >blending space < konstituiert haben, der infolge der MappingBeziehungen von >presentation space $<$ und >reference space $<$ angebahnt wird. Die beiden letztgenannten fungieren in Vorbereitung des >blending space $<$ als >input spaces<.

Dies ist das Kerngeschehen der >conceptual integration $<$ : Statt ein >blend $<$ als >Summe< oder >emergente Verschmelzung< darzubieten, wie es (vereinfacht ausgedrückt) in der Pionierzeit der >blending theory < oder auch in Harald Weinrichs Bildfeldtheorie suggeriert wurde, ${ }^{22}$ betont Brandt also nachdrücklich die Interaktion der >spaces $<$, die die Forschenden systematisch rekonstruieren und mit analytischen Zwischenstufen versehen müssen. Gerade weil die phänomenologische Gleichzeitigkeit der semantischen und ästhetischen Prozesse eine große Herausforderung darstellt, scheint die analytische Phasengliederung vielversprechend, zumal sie sich nicht auf die Introspektion beschränkt, sondern eine Anwendung an Beispielen zulässt.

Charakteristisch für den >blending space $<$ (4) ist der Ausdruck $>$ as-if-ness $<,{ }^{23}$ da hier der >reference space $<$ in einer Weise dargeboten wird, als würde er mit dem >presentation space< übereinstimmen. Es geht dabei wohlgemerkt nicht um prinzipielle oder vorgängige Ähnlichkeitsbeziehungen, sondern um dynamische Wechselbeziehungen zwischen den Domänen, welche das »als ob«bzw. »wie« erst evozieren.

Ebene 4 geht aus dem Mapping-Prozess ${ }^{24}$ zwischen den beiden >input spaces< (>reference space $<$ und >presentation space $<$ ) hervor: Unter Berufung auf Fauconnier versteht Brandt Mapping als Korrespondenzbeziehungen zwischen zwei vorübergehend gebildeten >mental spaces $<$, bei denen jedes Element von Input 1 eine Entsprechung in Input 2 findet. ${ }^{25} \gg$ The concept of counterparts presupposes structural comparability, on the basis of which elements in the source and in the target inputs may be fused or contrasted in a blend. $\ll^{26}$ Phänomenologische Ähnlichkeit und strukturelle Vergleichbarkeit sind demnach so weit wie möglich voneinander zu unterscheiden, um vergleichende Praktiken überhaupt >kleinarbeiten< zu können. Um die Korrespondenzbeziehungen nicht als starre Merkmalsliste, sondern dynamisch zu betrachten, kommen die Begriffe $>$ Schema $<$ und $>$ Szenario< zum Einsatz: Es wird ein Beziehungsgeflecht aus gemeinsamen oder anschlussfähigen Komponenten angelegt, weshalb der potenzielle Handlungsrahmen anhand von u.a. Agens, Patiens, Raum, Zeit und Zielausrichtung zu bestimmen ist. Brandt legt überzeugend dar, dass eine Aufzählung von Berufseigenschaften von Chirurgen wie Schlachtern nicht

\footnotetext{
22 Die Etablierung von Kategorien und die Inanspruchnahme zweier verschiedener Kategorien, wie z. B. Farbe und Form in der Vorstellung eines roten Balles, werden von Brandt im Unterschied zu etwa E. Sweetser und M. Turner nicht zum Blending bzw. zur >conceptual integration< gerechnet.

23 Brandt 2013, S. 260.

24 Das strukturell verwandte Verhältnis von Mapping-Beziehungen und der Herstellung von Analogien bedürfte noch einer genaueren Erforschung. Grundlegend für die Unterscheidung von tentativen Analogien einerseits und schlussfolgernden, weiterreichenden Analogien andererseits ist der Artikel von Roggenbuck, Simone: »Analogie als Ausgangspunkt für Vergleich und Klassifikation«. In: Michael Eggers (Hg.): Von Ähnlichkeiten und Unterschieden. Heidelberg 2011, S. 79-90.

25 Vgl. Brandt 2013, S. 269-270. Stellt man sich diese potenziellen Korrespondenzbeziehungen als Bündel gemeinsamer Merkmale vor, liegt es nahe, den Begriff der tentativen Analogie zu bemühen.

26 Brandt 2013, S. 269.
} 
weiterhelfe, da sie in diesem Beispiel eben nicht zur kommunizierten Bedeutung hinführe. Stattdessen arbeitet sie heraus, dass im Mapping-Prozess die unangemessene Behandlung und Herabwürdigung des Operierten, geradezu im Sinne einer entmenschlichten Produktverarbeitung, pointiert werde. Dabei wird auch die zeitliche Dimension in der Zweck- und Zukunftsorientierung der Handlung reflektiert: Während der Schlachter Fleisch von getöteten Tieren für die Nahrungsverwertung zerlegt, schneidet der Chirurg in die Körper von Menschen, die nach der Operation mit Narben weiterleben müssen. Erst auf Ebene 5, nämlich mittels der post-emergenten Bedeutung, trete die ethische Missachtung als zentraler Aspekt der >énonciation< hervor.

Anhand der Beschreibung des Mapping-Prozesses lässt sich ermessen, welche Bedeutungskomponenten bzw. temporäre >mental spaces < infolge eines Emergenzeffektes erst später hinzutreten, da sie weder in Input 1 noch in Input 2 angelegt waren. Gerade bei der Untersuchung einzelner Metaphern ist Ebene 5 besonders wichtig, weil so das Resultat des >blends < profiliert wird, das auf die Basisebene zurückwirkt, wodurch die Absicht der >énonciation< präzisiert werden kann. Im Fall des Surgeon/ Butcher-Beispiels träten laut Brandt auf diese Weise Vorwurf und Anklage ${ }^{27}$ prägnanter hervor: Besagter Chirurg habe den Patienten nicht in erster Linie dilettantisch behandelt, worauf sich die bisherige Forschung zu Blending bzw. >conceptual integration< geeinigt hatte, sondern jener Arzt habe auf unverantwortliche Weise die Konsequenzen seines Tuns ignoriert. ${ }^{28}$ Nicht die Annahme von Ähnlichkeiten beider Professionen sei grundlegend, ${ }^{29}$ sondern vor allem die (hier semiotische konturierte) Handlungsorientierung über einen bestimmten Zeitraum und die Relation von Agens und Patiens. Die Kritik am Chirurgen richte sich zudem an ein Gegenüber, das Empörung und Enttäuschung über den Ausgang der Operation möglichst teilen und Beistand leisten sollte.

Wird die semantische Analyse durch eine pragmatische gerahmt, scheint es möglich, sowohl Textsinn als auch performative Potenziale angemessen zu berücksichtigen. Die Introspektion der kognitiven Semantik ist für eine vorübergehende Dekontextualisierung geeignet, bedarf jedoch einer Rückeinbettung in den konkreten Zusammenhang von »Aussagehandlungen«, um sowohl spezifische als auch allgemeingültige Prozesse infolge des Mappings genauer bestimmen zu können.

\section{Beispielanalysen}

\subsection{Ein selbstermächtigendes Du}

Elmer Diktonius, einer der bekanntesten Vertreter des finnlandschwedischen Modernismus, verwendet in seinem Huldigungsgedicht an die bekannte Dichterin Edith

\footnotetext{
27 Vgl. ebd., S. 281.

28 Vgl. ebd., S. 241.

29 Es sind nicht die Metaphern, die zu allererst auf Ähnlichkeitsbeziehungen gründen (vgl. Lakoff u. Johnson 2003 passim), sondern die Analogien.
}

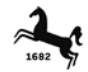


Södergran die innovative Metapher »Gedichtschnake(n)«. Diese werde ich ausgehend vom eben skizzierten Modell untersuchen.

\section{SÖDERGRAN}

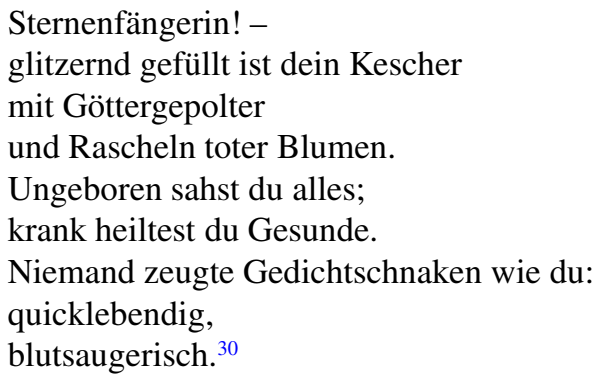

Statt einer Phänowelt wird ein textliches Universum konstituiert, innerhalb dessen Aussagen über Södergran und ihr Werk getroffen werden. Es gibt keine konkreten Requisiten, sondern nur eine vorgestellte Autorin und ihre erinnerte lyrische Produktion. Die >énonciation< betrifft demnach eine Ansprache bzw. eine literarisch vermittelte Kommunikation. Die Dichterin, vier Jahre vor der Veröffentlichung dieses Textes verstorben, wird von einem lyrischen Ich in einer fingierten Face-to-faceAnsprache angesprochen. Eben diese nicht-dialogische Kommunikation ist im >base space < anzusiedeln. Die Hinwendung zum Du beeinflusst auch die vorgestellte Gemeinschaft der Lesenden, von der angenommen wird, dass sie Schlussfolgerungen aus der Relation zwischen der gewürdigten Person und dem lyrischen Ich ziehen.

Die markante Einzelmetapher »Gedichtschnaken« im >blending space< bezieht sich auf das lyrische Werk Södergrans, wobei nach dem Schema PRODUKTION von einem Szenario des >Zeugungsaktes < und nicht etwa vom >Akt des Gebärens < die Rede ist. Im >presentation space < ist die konzeptionelle Einheit »Gedichtschnaken« verortet, d. h. > source < und >target $<$ werden in einer gemeinsamen Repräsentation dargeboten, dezidiert ein >Vergleich ohne wie $<$. Die Reihenfolge der Substantive legt eine Bedeutungshierarchie fest: Es handelt sich nicht um »Schnakengedichte«bzw. Gedichte, die Schnaken thematisieren, sondern um lyrische Texte, die etwas Schnakenhaftes haben - wohlgemerkt im Sinne von »as-if-ness « und nicht als vorgängige Ähnlichkeit.

Der bei der metaphorischen Bedeutungsgenerierung häufig anzutreffende Übertragungsweg vom >generic space < auf eine individuelle Einheit trifft auf dieses Beispiel insofern zu, als unspezifizierte »Schnaken« zum Vergleich mit dem lyrischen Werk just dieser Autorin herangezogen werden. Die Lesart kann selbstverständlich nur gelingen, wenn sich der Name der Dichterin in der Gedichtüberschrift den Lesenden sofort erschließt. ${ }^{31}$

\footnotetext{
${ }^{30}$ Liedtke, Klaus-Jürgen: Finnlandschwedische Literatur der Avantgarde. Gras und Granit. Aphorismen und Gedichte von Elmer Diktonius 1921-1954. Bd. 2, Münster 2014, S. 79; schwedischer Text: »SÖDERGRAN/ Stjärnfångerska! -/ din håv är glitterfull / av gudabrak / och döda blommors prassel. / Ofödd såg du allt; / sjuk botade du friska. / Ingen avlade diktmygg som du: / livslevande, / blodsugande «. Diktonius, Elmer: »Södergran«. In: Ders.: Stenkol. Helsingfors 1927, S. 30.

31 Oder der >reference space < bliebe leer und würde erst gefüllt, sobald Ebene 5 des Modells erreicht ist.
} 
Um den Mapping-Prozess ansatzweise rekonstruieren zu können, sind die Szenarien zu bestimmen und die Mapping-Korrespondenzen zu prüfen. Hierfür sind aus heuristischen Gründen allerdings »Schnake « im >presentation space « und »Gedicht« im >reference space $<$ zu verorten, wenn auch unter der Bedingung einer simultanen Wechselwirkung. Typische Handlungen von Schnaken sind das Fliegen, Kriechen und Verweilen, wobei sie den Betrachtern nur auf einem hellen Untergrund auffallen. Stechen und Blutsaugen oder die Verfolgung der potenziellen »Beute« (Schema JAGD) sind für diesen Akteur ebenfalls charakteristisch, wobei ein solcher Überfall die von Schnaken Verfolgten, trotz der geringen Größe des Insekts, wehrlos erscheinen lässt. Die genannten Tätigkeiten von Schnaken sind Gedichten gemeinhin nicht zuzutrauen, weshalb für das Mapping zunächst nur die feingliedrige Zusammensetzung der Insektenkörper und deren mögliche Beweglichkeit überprüft werden. Die graphische Erscheinung eines Insekts in vergrößertem Maßstab appelliert an Materialität und Layout des Gedichts. Mit dem länglichen Leib und den von diesen abzweigenden, feingliedrigen Extremitäten kann eine analogische strukturelle Beziehung zur Texträumlichkeit eines Gedichts in seinem Zeilenaufbau hergestellt werden.

Neben den als variabel dargebotenen Proportionen besteht eine fiktive Bewegungsoption: Gedichte könnten sich unter dem Einfluss einer solchen Dynamisierung über Papierseiten hin bewegen wie Insekten an einer Wand; auch zirkulieren publizierte Lyriktexte, und Leser können von imaginären und erinnerten Texten umschwirrt oder gar >infiziert< werden. Aber wie könnten derart mobilisierte lyrische Texte den Rezipienten Lebenssaft abzapfen? Hierauf ergibt sich erst dann eine Antwort, wenn man die Textsorte des Künstlergedichts berücksichtigt (s.u.).

Beachtet man zunächst die Einbettung in den unmittelbaren Ko-Text, gerät in den Blick, dass die Adjektive »quicklebendig « und »blutsaugerisch « sowohl die Gedichte als auch die gepriesene Figur charakterisieren könnten: Die gepriesene Autorin ist in einer kosmischen Welt mit einem Fanggerät unterwegs - auch sie jagt etwas und hat bereits »Göttergepolter« erbeutet. Dass die Gehuldigte sich in der Aufopferung für die große Kunst selbst verzehrte, wird als Interpretationsmöglichkeit durch den selbstreflexiven Bezug von »wie du« auf die beiden genannten Eigenschaften eröffnet. Eine märtyrerinnenhafte Inszenierung Södergrans wird aufgerufen, die bereits zu ihren Lebzeiten in der Literaturgeschichtsschreibung einsetzte, nicht zuletzt weil die Autorin an Tuberkulose verstarb. Mit dem Verb »zeugen « wird ein naturalisierter, männlich konnotierter Schaffensakt beschworen; dies unterstreicht den Ausnahmefall der einsamen weiblichen Schöpferposition. Als Christus-Inkarnation sei die emblematische Geniegestalt Södergrans über genderspezifische (Re-)Produktionsbedingungen gleichsam erhaben.

Im >blending space< werden mittels Mapping einige Korrespondenzen angeleitet, während andere sich nicht längerfristig etablieren, nachdem sie tentativ überprüft worden sind, wie z. B. die mögliche Analogie zwischen der Gliederung eines Schnakenbeins und der Metrik einer Gedichtzeile oder die Analogie zwischen Druckerschwärze auf der materiellen Außenseite des Gedichts und dunkler Chitinhülle des Insektenleibs. Es fällt auf, dass die von Lakoff/Johnson häufig beobachtete Tendenz, dass durch Metaphorisierungen abstrakte Sachverhalte konkret und anschaulich gemacht würden, auf diese Einzelmetapher kaum zutrifft: Zwar wird infolge des Map- 
pings die materielle Dimension des schwarz auf weiß gedruckten Textes betont, aber die Mehrdeutigkeit scheint eher vervielfacht als reduziert.

Das Mapping impliziert bestimmte Proportionen: Die Ausdehnung einer größeren Schnake könnte der eines kurzen Gedichts auf einer Papierseite entsprechen, wie etwa des kurzen Södergranporträts. Gerade eine solche Korrespondenz der Proportionen regt dazu an, auch die Aktionen Stechen oder Blutsaugen für den Gedichttext durchzuspielen, womit eine intertextuelle Dimension eröffnet wird. Die Gedichtlektüre mag sich für Rezipienten, vor allem aber für Dichter und Dichterinnen als eindringlich, sinnlich affizierend, ja sogar als unberechenbar und subversiv erweisen. Die Abfolge der Adjektive »quicklebendig, blutsaugerisch « wiese dann auf eine schnelle Inspiration mit darauffolgendem Energieverlust hin.

Die post-emergente Bedeutung der Gedichtschnaken im >meaning space $<$ festzulegen, leitet die Aufmerksamkeit auf die Textsorte. Kann man zu einer modifizierten Einschätzung des Gesamttextes gelangen, indem man sich auf den >base space < zurückbezieht? Sofern man Diktonius selbst - ausgehend von K. Hamburgers Auffassung vom lyrischen Ich - eine implizit autobiographische Stellungnahme zu bescheinigen wagt, kann Södergrans Vorbildfunktion genauer profiliert werden: Das lyrische Ich sieht sich befähigt und in der Lage, ein von Södergrans Gedichten konstituiertes Universum ermessen zu können. Diktonius' Porträt hebt das >ErgriffenWerden < hervor; die Huldigung beschwört eine vitalistische Eigendynamik der Texte jenseits einer rationalen Kontrollmöglichkeit durch die Rezipienten. Das Schaffen der mythisch überhöhten Dichterin stellt im Zuge dessen die Arbeiten anderer leicht in den Schatten. Die funktionelle Performativität wäre angesichts des Umstands, dass es sich um das einzige Porträt einer Künstlerin in Diktonius' Gedichtzyklus »Blixtporträtt « (Blitzporträts) ${ }^{32}$ handelt, wie folgt zu bestimmen: Mit der ambivalenten Insektenmetapher wird die beunruhigte Frage vermittelt, wie mit dem Phänomen der >anxiety of influence< angesichts eines weiblichen Genius umzugehen sei. Södergrans übermächtiger Einfluss mag irreversibel sein, so lautet die Prognose, und ihre Wirkmächtigkeit werde sich bis auf Weiteres fortsetzen.

Ziehen wir den finnlandschwedischen Ausgangstext heran, stellt sich heraus, dass hier lediglich von »Gedichtmücken« (»diktmygg«) die Rede ist. Wieso lediglich? Durch eine solche spontane Irritation wird ersichtlich, welche Folgen der soeben konstruierte >blending space < für weiterführende Deutungen hat. Das bisherige Interpretationsergebnis (aus Ebene 1 bis 5) stellt nämlich inzwischen den >semiotic base space< für anschließende Bedeutungsgenerierungen dar: Hat man zuerst die Übersetzung gelesen, will die Mücke nun klein und durchschnittlich erscheinen. Der >meaning space< stellt sich anders dar, obgleich das Szenario übereinstimmt. Der Mapping-Prozess zwischen den Inputs aktiviert Korrespondenzen; ${ }^{33}$ außerdem

\footnotetext{
32 Dieser Gedichtzyklus umfasst die Seiten 18-30 in Elmer Diktonius' Band Stenkol.

33 Dies hat sogar die Entscheidung des Übersetzers Liedtke begründet, eine Schnake statt einer Mücke zu wählen, neben seiner klanglichen Motivierung des »ausgreifenden« A-Lautes (siehe Mailwechsel mit Klaus-Jürgen Liedtke vom 31.8.15 u. 14.9.15). Die bestehenden dialektalen Schwankungen in der Verwendung von »Schnaken« und »Mücken« im deutschsprachigen Raum können hier nicht berücksichtigt werden.
} 
setzt das Verhältnis der Alterität zwischen Übersetzung und Ausgangstext eine Überprüfung von Differenzen in Gang.

Ein Gedicht als Mücke zu betrachten (>as-if-ness<, s.o.), bringt statt Pathos Alltäglichkeit ins Spiel, wenigstens im Deutschen. Der erfahrene Übersetzer KlausJürgen Liedtke entscheidet sich für die Schnake, obgleich er »diktmygg « im Schwedischen mit dem doppelten spitzen i- und ü-Laut als prägnant auffasst. ${ }^{34}$ Für das oben erwähnte Proportionenverhältnis von Insekten- und Textkörper ist in entscheidender Weise der ausgreifende Klang von »Schnake« wesentlich. Verglichen mit der kurzen Aussprache von »Mücke« erscheint das Schnakenkonzept räumlich expansiver, vor allem, wenn man den Kontrast infolge des Mappings durch weitere Merkmalsüberprüfungen auffächert. In das Schema der PRODUKTION fügt sich nun das Hervorbringen von Lauten ein: Nur die Mücke ruft die akustische Wahrnehmung auf, denn ein irritierendes Sirren wird der gravitätischen Schnake eher nicht unterstellt. Ebenso fällt das kollektive Auftreten der Mücke im nivellierenden Schwarm auf, weshalb die Schnake aus der Gegenüberstellung der jeweiligen Mapping-Prozesse als anspruchsvollere Solistin hervorgehen mag. Mithin bietet das filigrane Einzelwesen eher Korrespondenzen zur einsam erschaffenden, einzigartigen Dichterin als ein kollektiv auftretendes Kleininsekt. Der >meaning space〈 für die »Gedichtmücken« leitet die Schlussfolgerung an, dass die allgegenwärtigen Kürzesttexte sowohl stimulierende als auch irritierende Bestandteile der Lebenswelt sind, obwohl sie auf eine kosmische Erzeugerin zurückgehen. Der Literaturwissenschaftler Bill Romefors spricht in seiner Interpretation von einer fortbestehenden Aktivierung der Lesenden, die sich als körperlicher Effekt manifestiere. ${ }^{35}$ Die Modernismus-Expertin Kristina Malmio weist auf das wiederkehrende stilistische Verfahren in Diktonius' Gedichten hin, großes Pathos (»gudabråk«) gegen das banale Detail auszuspielen, eine textimmanente Spannung zwischen den Proportionen als Rahmungen, die auch bei der Erwähnung des Blumenraschelns ${ }^{36}$ im Unterschied zum Gepolter des Alls evoziert wird. ${ }^{37}$ Auch die übrigen zwölf Porträtgedichte bestätigen verwandte dynamische Topoi, die ein Szenario eines rasanten Zooms begründen, beispielsweise in einer Bewegung von der Totale zum Detail ${ }^{38}$ oder von einer Ineinander-Verschränkung von Pathos und Banalität als einer avantgardistischen Verschmelzung von High and Low. ${ }^{39}$

Durch das kontrastive intertextuelle Zusammenspiel beider Fassungen tritt eine weitere Modellierung der Huldigung Södergrans hervor: Das lyrische Ich nimmt

\footnotetext{
34 Mailwechsel mit Liedtke, ebd.

35 Die genaue Formulierung lautet: »den södergranska dikten fortsätter att aktivera läsarna genom en fysiskt påtaglig bild: det är >diktmygg< som sticks. « (dt. »Södergrans Gedichte werden die Leser weiterhin in Aktivität versetzen durch dieses körperlich konkrete Bild: Es sind >Gedichtmücken<, die stechen. «) (Romefors, Bill: Expressionisten Elmer Diktonius. En studie i hans lyrik 1921-1930. Helsingfors 1978, S. 152). Den Hinweis auf Romefors verdanke ich Kristina Malmio, siehe Mailwechsel vom 1. bis 4.2.2016.

36 Die Verwendung des Oxymorons (hier: gefüllt und leer, vorgeburtlich und weise, krank und gesund) würde eine genauere Untersuchung verlangen, als sie hier geliefert werden kann.

37 Mailwechsel mit Malmio vom 1. bis 4.2.2016.

38 Diktonius: Stenkol. S. 19.

39 Ebd., S. 21. Der Dichter Gunnar Björling tritt als Verwandter Södergrans auf, weil er ebenfalls auf die Jagd geht. Er erbeutet einen Käfer, eine Karotte, eine Perücke sowie Jazz (vgl. ebd., S. 21).
} 
eine Selbstpositionierung vor und spricht aus dem Kreis der Anhänger an die Dichterin, mit einer simultanen Hinwendung an die Lesenden, die wiederum eingeladen sind, sich mit der im Text angebotenen Subjektposition Södergrans zu identifizieren - im Sinne einer Hinführung zum Werk über eine eingängige identifikatorische Lesart. Das sich in der Übersetzung von Liedtke entfaltende Pathos intensiviert die Apotheose, und die Aufopferung Södergrans für die Kunst wird in der deutschen Übersetzung etwas stärker als im schwedischen Text pointiert. Liedtkes Fassung unterstreicht somit auch die nobilitierende Selbstcharakterisierung des lyrischen Ich, wobei sich mit größter Wahrscheinlichkeit der Einfluss der wirkmächtigen Södergran-Rezeptionsgeschichte auf die Gedichtübersetzung geltend macht.

Als Resultat der beiden Lektüren ist festzuhalten, wie schwer es fällt, wieder hinter eine plausible Interpretation zurückzugehen, wie sie eine tragfähige Übersetzung darstellt. Die »Gedichtmücke« behält eine innere Ambivalenz, wenn die Lesenden mit der »Gedichtschnake« Bekanntschaft gestiftet haben. Was man gemeinhin vage Anthropomorphisierung nennen würde, ist in beiden Gedichtfassungen als Neuperspektivierung eines Textinsekts dargeboten, das Imaginationen über die mediale Materialität und eine textsortenspezifische Kräftedynamik anleitet.

Das Beispiel belegt, dass das Kraftzentrum von Metaphern tatsächlich im >blending space < anzusetzen ist. Es lässt erahnen, dass man den mutmaßlichen MappingProzessen sowie selbst den persönlichen Zwischenprodukten der Imagination nur ansatzweise rekonstruierend $>$ hinterherforschen $<$ kann. ${ }^{40}$ Vielmehr findet eine mitgestaltende Aktualisierung statt, die verrät, dass sich die Conceptual-Integration-Forschung rezeptionsästhetischer und -geschichtlicher Fragen annehmen muss, wenn sie für eine literaturwissenschaftliche Anwendung ausgelegt wird.

\subsection{Sabotierte Gemeinsamkeit}

Mit dem folgenden dramatischen Dialog begeben wir uns in die symbolistische Bergwelt von Henrik Ibsens Wenn wir Toten erwachen (1899). Die Analyse soll demonstrieren, wie wichtig die Intentionalität der Sprechenden, hier der Dramenfiguren, für die Bedeutungsgenerierung im Rahmen der >conceptual integration< ist: »the inputs are viewed as mental spaces set up for a specific purpose, with content that pertains specifically to the topic of conversation $\ll .{ }^{41}$ Das Gespräch zwischen dem ehemaligen Künstlermodell Irene und dem Bildhauer Arnold Rubek problematisiert ein potenziell gemeinsames >blend . Hierbei wird Einblick in die figurenspezifische Interpretation gegeben - abgeleitet aus den jeweiligen >input spaces $<$ und zugleich bezogen auf das von den beiden Akteuren verhandelte Mapping und ihren >blending space<.

Der Schauplatz des Dialogs ist im Lesedrama eine Berglandschaft mit einem Bach und sommerlichen Pflanzen. In den unterschiedlichen Bühneninszenierungen wird diese Anweisung mit verschiedensten Kulissen und entsprechenden Phänowelten umgesetzt, was die gesamte Bühnensemiotik entscheidend prägt und damit auch Vorannahmen über die Modi von Referenz und Repräsentation mitteilt. Als einziges

40 Vgl. Kövecses: Metaphor. S. 268; vgl. Weinrich, Harald: Sprache in Texten. Stuttgart 1976, S. 286.

41 Brandt 2013, S. 265. 
Ibsen-Drama ist Wenn wir Toten erwachen in einem geographisch benannten Exterieur verortet, was den Status der Umgebung jedoch nicht vereindeutigt, da die Möglichkeit eines durchgängig allegorischen Verständnisses besteht. Indessen trägt auch die literaturhistorische Einordnung großen Anteil daran, welche Zeichenbezüge in der Figurenkommunikation von den Rezipierenden jeweils aktiviert werden. Eine symbolistische Aufführung könnte im Extremfall die allegorische Deutung so hoch ansetzen, dass beispielsweise die Referenz auf die im Wasser schwimmenden Blätter geschwächt wäre. In meiner Deutung werde ich indessen von konkreten Requisiten ausgehen und sowohl den Bach als auch die Blätter unabhängig vom situativen Stilisierungsgrad als materiell vorhandene Einheiten annehmen.

Das Gespräch der Figuren findet anlässlich eines Wiedersehens des ehemaligen Paares nach einigen Jahren der Trennung statt: Irene, die zuvor ihr gesamtes Leben auf die Beziehung zu Arnold Rubek ausgerichtet hatte und sich nach ihrem Zusammenbruch in psychiatrischer Obhut befindet, trifft ihren früheren Mann während eines Bergurlaubs wieder. Auf einem Spaziergang unterhalten sich die beiden über ihre zurückliegende Zusammenarbeit. Dabei kommt auch Rubeks einstige Abweisung Irenes zur Sprache, als diese sich gegen ihre leblose Rolle als Modell-Muse aufgelehnt hatte. Irene versucht einen Neuanfang, indem sie sich um einen >konzeptionell-sprachlichen< Konsens bemüht. Im Folgenden kommentieren die Figuren die Phäno- und Diskurswelt zugleich:

Irene: [...] Verscheuchen wir all die trüben und schweren Gedanken. (Sie pflückt die Blütenblätter einer Gebirgsrose und streut sie in den Bach.) Schau, Arnold - da schwimmen unsere Vögel.

Rubek: Und was sind das für Vögel?

Irene: Siehst du denn nicht? Das sind doch Flamingos - rosenrote Flamingos.

Rubek: Flamingos schwimmen nicht. Die waten nur.

Irene: Nun, dann sind es eben keine Flamingos. Dann sind es Möwen.

Rubek: Ja, das schon eher - Möwen mit roten Schnäbeln könnten es sein. ( $E r$ pflückt breite grüne Blätter und wirft sie ins Wasser.) So, und jetzt schicke ich meine Schiffe hinter ihnen her.

Irene: Aber es dürfen keine Vogelfänger darauf sein.

Rubek: Nein, Vogelfänger sind nicht an Bord. (Sieht sie lächelnd an.) Weißt du noch, den Sommer, da unten am See? Als wir so wie hier am Bachrand saßen, draußen vor dem kleinen Bauernhaus?

Irene: Ja - am Samstagabend - wenn wir mit unserer Arbeit für diese Woche fertig waren und hinausfuhren an den See -

Rubek: Und den Sonntag über draußen blieben -

Irene (mit einem bösartigen Funkeln im Blick). Es war nur eine Episode, Arnold.

Rubek (der tut, als habe er es nicht gehört). Da ließest du auch Vögel im Bach schwimmen. Es waren Wasserlilien [sic, weiße Seerosen, AW], die du Irene: Weiße Schwäne waren es.

Rubek: Jaja, Schwäne - das meinte ich auch. Und an einem der Schwäne, das weiß ich noch, befestigte ich einmal ein großes rauhes Blatt. Ich glaube sogar, es war ein Sauerampferblatt - 
Irene: Und das war dann das Boot des Lohengrin - mit dem Schwan davor.

Rubek: Dieses Spiel machte dir so viel Freude, Irene.

Irene: Und du - du sagtest, ich sei der Schwan vor deinem Boot.

Rubek: Hab ich das gesagt? Ja, das mag wohl sein. (In das Spiel vertieft, eifrig.)

Sieh doch nur, wie deine Möwen den Fluß hinunterschwimmen!

Irene (lachend). Und alle deine Schiffe stranden.

Rubek (indem er weitere Blätter auf die Reise schickt). Ich habe noch Schiffe genug zur Verfügung. (Sieht den schwimmenden Blättern nach, macht einzelne wieder flott und sagt nach einiger Zeit.) Übrigens, Irene - das kleine Bauernhaus da am See - das habe ich gekauft. ${ }^{42}$

Um die Erinnerung an die damalige Zweisamkeit zu aktualisieren, beginnt Irene ein Spiel mit Requisiten aus der Natur: Sie lässt abgepflückte Blütenblätter auf der bewegten Wasseroberfläche treiben und erklärt diese zu »unseren Vögeln«, um Rubek mit Hilfe des Spiels in die gemeinsame Vergangenheit zurückzuversetzen. Das Gespräch über das Spiel widmet sich implizit zugleich der Paarkonfiguration.

Die Figuren verwenden unterschiedliche Objekte: Irene wählt zunächst rote Blätter (im >reference space $<$ ) für exotische Flamingos (im >presentation space $<$ ), während sie in der Vergangenheit weiße Blätter als Zeichen für einen Schwan bzw. Schwäne eingesetzt hatte. Rubek ist für die grünen Blätter zuständig, die im Bergbach den Blütenblättern hinterhertreiben sollen.

Dass die Möwen ins Spiel gebracht werden, ist Rubeks Anwendung der Kategorien >Schwimmfähigkeit< und Farbe zu verdanken. Es geht ihm aber nur vordergründig um das ornithologische Etikett der Watvögel, denn seine Ausweichmanöver sind spätesten im >meaning space< argumentativ nicht mehr plausibel. Dabei unterstreicht

\footnotetext{
42 Ibsen, Henrik. Wenn wir Toten erwachen. Dramatischer Epilog in drei Akten. Aus dem Norwegischen übertragen und mit einem Nachwort von Hans Egon Gerlach. Stuttgart 1965, S. 49-50; norwegischer Text: Ibsen, Henrik: Når vi døde vågner. Hovedtekst, 1.utg. 1899. In: Henrik Ibsens skrifter. Historisk-kritisk utgave, Hg. Janss, Christian, Stine Brenna Taugbøll u. Ellen Nesheim Wiger, Oslo 2010, S. 169-288, hier S. 256-258; zitiert nach https://www.ibsen.uio.no/DRVIT_NVINVht.pdf (zugänglich 10.7.2019), S. 51-52. »IRENE [...] Lad os ryste det dybe og det tunge af os. (plukker bladene af en fjeldrose og strør dem i bækken) Sé der, Arnold. Der svømmer vore fugle. / PROFESSOR RUBEK Hvad er det for fugle? / IRENE Kan du ikke sé det? Det er jo flamingoer. For de er rosenrøde. / PROFESSOR RUBEK Flamingoer svømmer ikke. De bare vader. IRENE Så er det ikke flamingoer da. Det er måger. / PROFESSOR RUBEK Måger med røde næb kan det være, ja. (plukker brede grønne blade og kaster dem ud) Nu sender jeg mine skibe ud efter dem. / IRENE Men der skal ikke være fangstmænd ombord. / PROFESSOR RUBEK Nej der skal ingen fangstmænd være. (smiler til hende) Kan du huske den sommer vi sad sådan udenfor det lille bondehuset ved Taunitzer See? / IRENE nikker Lørdags aftenerne, ja, - når vi var færdige med vort arbejde for ugen - / PROFESSOR RUBEK - og rejste ud med jernbanen. Og blev derude søndagen over - / IRENE med et ondt, hadfyldt glimt i øjet Det var en episode, Arnold. / PROFESSOR RUBEK som om han ikke hører Da lod du også fugle svømme i bækken. Det var vandliljer, som du - / IRENE Hvide svaner var det. / PROFESSOR RUBEK Jeg mente svaner, ja. Og jeg husker, at jeg fæsted et stort loddent blad til en af svanerne. Det var endda et skræppeblad - / IRENE Så blev det til Lohengrins båd - med svanen foran. / PROFESSOR RUBEK Hvor glad du var i den leg, Irene. / IRENE Vi legte den ofte om igen. / PROFESSOR RUBEK Hver eneste lørdag, tror jeg. Hele sommeren udover. / IRENE Du sa', at jeg var svanen, som trak din båd. / PROFESSOR RUBEK Sa' jeg det? Ja, det kan godt være. (optaget af legen) Nej sé bare, hvor mågerne svømmer nedover elven, du. / IRENE lér Og alle dine skibe strander. / PROFESSOR RUBEK sætter mere løv i bækken Jeg har skibe nok i behold. (følger løvet med øjnene, stikker flere blade ud og siger lidt efter) Du Irene, - jeg har købt det lille bondehuset ved Taunitzer See.«.
} 
seine Einschätzung, dass der rote Möwenschnabel die Farbe der Blütenblätter angemessen vertrete, sein Deutungsvorrecht eines empirisch-positivistischen wissenschaftlichen Codes. ${ }^{43}$ Zugleich gibt er damit bekannt, welche Analogiebeziehungen sein subjektives Mapping fundieren.

Mit den Möwen und Vogelfängerschiffen wird von Rubek ein gänzlich anderes Szenario als der liebliche Bachlauf mit rosigen Ziervögeln beschworen: Die Meeresvögel würden laut Rubek von Fängerschiffen verfolgt, seien also eine Beute. Diesem Bruch entspricht der abrupte Ortswechsel vom Gebirge in eine Küstenlandschaft. Es handelt sich außerdem um ein Jagdszenario, das mit der Erwähnung der Vielzahl und Größe der grünen Blätter - als Einheiten seiner Flotte - Rubeks Besitz und hegemoniale Position betont. Irene wendet sich ausdrücklich gegen das Jagdspiel: Mit dem Lohengrin'schen Gespann ${ }^{44}$ aus Schwan und nachfolgendem Boot wird von ihr stattdessen das Szenario einer Liebesfahrt entworfen, das von alltagsrealistischen Wahrscheinlichkeiten (wie sie der norwegische Vogelfang auf dem Meer plötzlich vergegenwärtigt) entbunden ist. Bezeichnenderweise ändern sich die figurenspezifischen Requisiten im Dialogverlauf, seitdem Rubek die Zeitstufe der Vergangenheit thematisiert, da die weiße Seerose und das Ampferblatt als Gegenstände der Erinnerung zum Einsatz gelangen und mit Inanspruchnahme einer weiteren Zeit- und Bewusstseinsstufe ein zusätzlicher >mental space< eröffnet wird. Rubek versucht sich also an einer intersubjektiven, d.h. gemeinsamen imaginären Aktualisierung des erinnerten Spiels, bestreitet jedoch, das Lohengrin-Gespann als bildspendendes Konzept (source) jemals angeregt zu haben. Die Spielszene in der Gegenwart gibt ihm sogar die Möglichkeit, einen Themenwechsel einzuleiten, der sich vollzieht, als wieder von Möwen die Rede ist, was die Entzauberung des potenziellen gemeinsamen Moments herbeiführt. Irene hält abschließend fest, dass Rubeks Szenario keine erfolgreiche, zum Abschluss gelangende Handlung ermöglicht.

Mit den eingesetzten Verben sind unterschiedliche Aktivitätsgrade verbunden: Das autonome Schwimmen der Flamingos wird durch die selbständige Bewegung der Möwen abgelöst, die indessen die Folgebewegung der Jäger bedingt. Der Schwan, der Lohengrins Nachen zieht, entscheidet in Wagners Libretto über die Fortbewegung des Bootes und das Schicksal eines Passagiers. Das Mapping ist bereits angedeutet in den Bezügen, die zwischen Farbe, Form, Aktivität sowie in der Reihenfolge von Agens/Patiens hergestellt werden. Rubek überprüft widerstrebend Analogien, während Irene gezielt auf die symbolische Aufladung und die gemeinsame Code-Verwendung hinarbeitet. Hieraus ergibt sich notwendigerweise, dass Irene einen >blending space< erzeugt, in welchem Blütenblätter als märchenhafte Vögel zu dekodieren sind, während Rubek seine grünen Blätter wie in einer Modelllandschaft einsetzt. Die Requisiten bleiben aus seiner Sicht materielle Spielfiguren im >semiotic base space $<$, sie verweisen allenfalls auf das vergangene Spielritual und sind nicht Bestandteil eines von ihm vorgestellten eigengesetzlichen Universums. Ibsens Spielszene verblüfft durch ihre quälend-komische Demontage

\footnotetext{
43 Vgl. »Analogy Blends«, Brandt 2013, S. 420.

44 Vgl. Wagner, Richard: Lohengrin, Textbuch mit Varianten der Partitur, hg. Egon Voss. Stuttgart 2001, passim.
} 
einer berühmten Konzeptmetapher von Lakoff/Johnson: »Love is a journey. $\ll^{45}$ Die beiden Figuren sind, wie die konkreten und imaginären Akteure und Objekte, nachweislich nicht in der Lage, eine bildhafte gemeinsame Reise durchzuführen, sowohl Metaphernsynchronisierung als auch Liebesbeziehung sind nachdrücklich gescheitert.

Auf der post-emergenten Ebene lässt sich erkennen, dass das Scheitern der Kommunikation auf die unterschiedlichen Zeichencodes der Figuren zurückgeführt werden muss. Irenes intertextueller Horizont sorgt für ihr gleichnishaftes Sprechen als würde markiert, dass jeder Ausdruck als symbolisches Zeichen fungieren soll. Im Voraus besteht eine tragische Ironie darin, dass das Lohengrin-Motiv ohnehin auf Liebesverzicht hinweist. Irene bemüht sich, den Liebescode auf die Zeitstufen Vergangenheit, Gegenwart und Zukunft zu beziehen, als wollte sie Kontinuität wiederherstellen. Rubek bevorzugt dagegen die metonymische Vergewisserung, also eine auf Indexikalität basierende Zeichenrelation. Sein Ignorieren des symbolischen Codes ist besonders deutlich in der Betonung von Quantität und ökonomischer Materialität: Die Blättermenge bildet am Ende des Dialogabschnitts recht beliebige Einheiten, und die Immobilie am See erscheint als plumpe Annexion, die mit der nostalgischen Spielszene Irenes unvereinbar ist. Mit dem Wissen im Nachhinein scheint die Regieanweisung für Rubek, der »tut, als habe er es nicht gehört « eine Quintessenz der Code-Kollision zu sein. Weder kann noch will sich der Künstler Rubek in Irenes Zeichen und Codes hineinversetzen, seine Empathieverweigerung ist entweder absichtsvoll oder einem Mangel an Kompetenz geschuldet. ${ }^{46}$

Der Untertitel dieses letzten Ibsen-Dramas lautet »Epilog «, was zusätzlich eine metadramatische Kommentierung eröffnet. Bildete der sogenannte Epilog einen Kommentar zu Ibsens abgeschlossener Serie von Gesellschaftsdramen, deutete sich eine skeptische Einschätzung über das Vermögen von Literatur und Kunst an, Antworten auf existenzielle Fragen geben zu können. Rubeks offizieller und ökonomischer Erfolg diskreditiert seine ehemaligen hohen Ziele, während die idealistische Position in Irenes Gestalt nun entrückt oder gar wahnhaft erscheint. Das Gespräch des Paares selbst gibt sich ebenfalls als Epilog, insbesondere durch die Bemühung des leitmotivischen Begriffs »Episode « für einen unwiderruflich abgeschlossenen biographischen Abschnitt. Die Relation der Einheiten >Epilog-Drama Wenn wir Toten erwachen zu Ibsens Gesellschaftsdramen< einerseits und >scheiterndes Figurengespräch zu Epilog-Drama Wenn wir Toten erwachen< andererseits wird gängigerweise Strukturanalogie genannt und entspringt einer metonymischen Grundannahme. Es zeugt von der Macht der literaturhistorischen Tradition, diese Wissenseinheiten in den >meaning space< oder bereits in den >base space< einzubringen und die Lesart des Künstlerdramas als durchgängige Wahrnehmungsvorstrukturierung zu bemühen.

Blickt man von Ebene 5 auf die Basiskoordinaten des Dialogs zurück, tritt der wichtige Anteil der Performanz bei der Bedeutungsgenerierung hervor. Je nach Inszenierung ergibt sich ein spezifischer >meaning spaceく, selbst bei weitreichenden semantischen Übereinstimmungen sind markante Differenzen zu erwarten, wie an

45 Vgl. Lakoff u. Johnson 2003, S. 44-45.

46 Hiermit ergibt sich eine markante kräftedynamische Korrespondenz zu Rubeks Künstlerkrise. 
den extrem vielfältigen Ibsen-Inszenierungen studiert werden kann. Die Interaktion des Paares auf der Bühne entscheidet maßgeblich darüber, wie die Rezipienten etwa die sprachphilosophische, kunstthematische oder ästhetische Repräsentationskritische Reflexion des Dialogs gewichten. Selbstverständlich haben auch die figurenspezifische Darstellung der Affekte in der psychologischen Auseinandersetzung, d.h. die Rollenbesetzung und schauspielerische Leistung großen Anteil daran, wie sich der >presentation space< der Bühneninszenierung auf den Konflikt zwischen dem idealistischen und dem realistischen Code im >reference space< auswirkt. Nicht $\mathrm{zu}$ vergessen ist die elementare Grundsatzentscheidung einer jeden Theaterinszenierung, einen >ground < und >semiotic base space< zu entwerfen. Dies führt auch zur eingangs formulierten Frage der Bühnengestaltung zurück.

Für die Dramenanalyse erweisen sich somit >semiotic blends < als zentral, da in diesen der > presentation space < zeichenhaft für den >reference space< einsteht. Zu den >social semiotic blends $<$, welche auf die Deutung der Aufführungssituation großen Einfluss haben, rechnet Brandt in ihrer innovativen Typologie der Integrationsarten ${ }^{47}$ >ludic blends $<$ und >performative blends $<$. (Letztere bezieht Brandt allein auf fixierte Rituale, sie können sich daher kaum für eine Inszenierungsanalyse entfalten.) Die >ludic blends < werden erwartungsgemäß bei einem Spiel im Spiel exemplarisch umgesetzt, sind entsprechend metadiskursiv angelegt und entfalten sich auf eine Weise, die Reflexionsmöglichkeiten über Theatralität eröffnet. Nichtsdestotrotz bleibt diese Projektion recht vage, wenn sowohl das Spiel im Spiel als auch die theatrale Darbietung insgesamt durch folgende Definition erfasst sind: »In ludic integrations, the cognizer is interacting with something as if it were something else. $\ll^{48}$ Auch das Merkmal »enactment of the pretend scenario $\ll^{49}$ trifft auf die ausgewählte Spielszene und die gesamte Aufführungssituation gleichermaßen zu, obgleich es immer noch lohnend erscheint, deren »base space«, die beanspruchten Szenarien, die Bildung von post-emergenter Bedeutungen vergleichend aufeinander zu beziehen - gerade weil es sich um eine wechselseitige Kommentierung handelt. Irene und Rubek unternehmen den Versuch, das damalige, erinnerte Spiel mit einem gegenwärtigen zu verschränken, was misslingt, weil die individuellen Deutungen der biographischen Zeitstufen voneinander abweichen. Den Zuschauern bietet sich nichtsdestotrotz ein Moment der sogenannten Zeitlosigkeit (»time out of time $\ll^{50}$ ), was Brandt als $>$ cotemporality integration < begreift: »the represented entities come to virtually co-exist in one scenario, constructed as a narrative unfolding in chronological and ahistorical time. $\ll^{51}$ Ein solches Verfahren verweist ebenfalls auf verallgemeinerbare Strategien eines auf Imaginationen gründenden Schreibens (oder Sprechens), wenn nicht gar auf Grundbedingungen der Fiktion, die mit Erinnerung und Zeitlichkeit befasst ist. Weitaus wichtiger erscheint mir, dass eine unüberbrückbare Isolation und Kommunikationslosigkeit der Figuren mittels einer unmöglichen Synchronisierung im >blending space< performativ nachvollzogen wird. Anhand des > virtual identity

\footnotetext{
47 Vgl. Brandt 2013, S. 406-407.

48 Vgl. ebd., S. 415.

49 Vgl. ebd., S. 415.

50 Ebd., S. 138.

51 Ebd., S. 416.
} 
blends ${ }^{52}$ lässt sich weiterhin durch die konzeptuelle Integrationstheorie begründen, warum ein gemeinsames >blend < der Figuren hier ausgeschlossen ist, denn die Referenz (Blütenblatt) wird nur momenthaft von Irene mit der Präsentation (Vogel) identifiziert, bevor auch schon >presentation space < und >reference space< wieder in Frage stehen: »Nun, dann sind es eben keine Flamingos. « Als kleinster gemeinsamer Nenner bleibt am Ende des Gesprächs die Analogiebildung eines doppelten illusorischen Spiels selbst übrig, auf der Bühne des Theaters und des Lebens. Rubek erhebt dabei die tentativen Analogiebeziehungen als Bestandteile des Mappings zu einem eigenen Thema. Zudem führt der Dialog metadiskursiv vor, dass eine Benennung von Differenzen und Übereinstimmungen stets aus den bedeutungserzeugenden Handlungen des Kontrastierens und Angleichens hervorgeht.

\section{Komparatistische Positionsbestimmung}

Für beide Fallbeispiele waren intratextuelle wie intertextuelle Beziehungen von wesentlicher Bedeutung, gleichermaßen zeichnete sich die Notwendigkeit ab, rezeptionsästhetische und -historische Aspekte in die Überlegungen miteinzubeziehen. In Beispiel 1 stand der folgenreiche Austausch zwischen einer Gedichtübersetzung und ihrem Ausgangstext im Zentrum: Die erste Lesart der übersetzten Fassung ging dabei in den >semiotic base space< ein, der das Fundament für die nachfolgende Interpretation des schwedischen Ausgangstextes bot. Eine zusätzliche Pointe besteht darin, dass sich der Übersetzer Liedtke auf eigene intertextuelle Erfahrungen im Umgang mit modernistischer und schriftthematischer Lyrik beruft - nicht zuletzt bei der Übertragung von Insektenmotiven. ${ }^{53}$ Liedtke hat aufgrund des ihm bekannten Textkorpus einen eigenen Referenzrahmen etablieren können, der eine subjektive Grundlage für jede weitere Übersetzung ausmacht. Auf prägnante Weise zeichnet sich hier eine komparatistische Kernfrage zu intertextuellen Wissensrahmungen ab.

In Beispiel 2 wendet eine Dramenfigur im Gespräch einen bekannten, in sich bereits symbolträchtigen literarischen Text für eine Wahrnehmungsstrukturierung an, durch die der Gesprächspartner dazu animiert werden soll, einen bestimmten Code $\mathrm{zu}$ verwenden und eine nostalgische Haltung einzunehmen. Darüber hinaus widmet sich der Figurendialog - auf der Metaebene - dem Thema, ob und wie ein subjektiver >blending space $<$ und ein ad-hoc-Blend intersubjektiv kommuniziert werden können. Eine post-emergente Bedeutung auf letzter Stufe kommt nicht zustande, was aus Rezipientensicht das Scheitern der Figurenrelation hervorhebt. Die Koordinaten im »base space« wurden dabei als entscheidend für die konkrete Inszenierung dieses Ibsen-Dramas erachtet. Aus Brandts Typologie konzeptueller Integrationen wurden >ludic blends< und >co-temporality blends< herangezogen, um einen Bogen zu Aufführungen zu schlagen, bei denen ein >blending space $<$ mit bühnentechnischen und medienspezifischen Mitteln angeleitet werden kann.

Im ersten wie im zweiten Beispiel kamen sprachliche und konzeptuelle Faktoren in einer teilweise komplexen Interaktion zum Tragen: Es manifestierten sich

\footnotetext{
52 Vgl. ebd., S. 145-150 u. S. 416-420.

53 Siehe Mailwechsel mit Liedtke vom 31.8.15 u. 14.9.15.
} 
unterschiedliche mögliche Bedeutungen trotz übereinstimmender konzeptueller Metaphern bzw. Szenarien. Als ein Nebenergebnis trat hervor, dass oft tentative Analogiebildungen den Mapping-Prozess anleiten. Der Begriff der Analogie wird von Brandt eher vermieden, ist aber meiner Einschätzung nach in den >Korrespondenzen $<$ und >Entsprechungen $<$ (>counterparts $<$ ) stets präsent. Daran schließt sich die Frage an, ob nicht eigentlich Analogienbildungen das jeweilige tertium comparationis von Vergleichen motivieren. Im eingangs verwendeten Zitat von Epple und Erhart ist interessanterweise von einer »Bewegung von Gemeinsamkeiten und Unterschieden« (s.o.) die Rede, die vom tertium comparationis angeregt würden. In Erweiterung dessen veranschaulichen die beiden Fallbeispiele, dass die tentativen Analogiebildungen wiederum die Wahl des tertium comparationis beeinflussen.

Die Perspektive der >conceptual integration< lässt sich nutzen, um komparatistische Vorgehensweisen selbstkritisch zu hinterfragen. Die Forschenden sind - wie auch die AutorInnen und Rezipierenden - bekanntlich während des Vergleichens stets selbst produktiv und mit (Vor-)Entscheidungen der Interpretation befasst, die stets auch mit wertenden Handlungen einhergehen. Gerade die konzeptuelle Koproduktion der Forschenden wird oft zu wenig beachtet: Sie stellt eher ein Eingreifen dar als eine begleitende Kommentierung. Ohne einem >cognitive turn< das Wort zu reden, verlangt die konzeptuelle und sprachliche, gestalterische Mitwirkung eingehendere Untersuchungen, nicht zuletzt weil sich durch die systematische Analyse der vergleichenden Tätigkeiten $u$ nd der Koproduktion auf plastische Weise Einblicke in das wissenssoziologische Profil komparatistischer Forschungen ergeben.

Gemeinhin anerkannten Vergleichsparametern und konventionell angewandten Kategorien ist mit wachsamer Kritik, wenn nicht Skepsis zu begegnen. Statt mit zugeschriebenen Eigenschaften zu operieren, die ohnehin erst während der vergleichenden Tätigkeit konstituiert werden, sollten die Mapping-Beziehungen im Einzelnen aufgearbeitet werden. Die Mapping-Beziehungen veranschaulichen, dass das Besondere und das Allgemeine nicht isoliert voneinander betrachtet werden können, weil sie eben miteinander verzahnt bleiben. Dennoch kann die Aufforderung des Komparatisten Stefan Willers, die allgemeine Dimension des Vergleichs wieder intensiver zu erforschen, ${ }^{54}$ bezogen auf die Conceptual-Integration-Forschung insofern umgesetzt werden, als diese verlangt, die Vorannahmen über das sogenannte Allgemeine und Besondere in der komparatistischen Arbeit zunächst einmal offenzulegen. Hierbei besteht eine der Herausforderungen darin, vergleichende Tätigkeiten auch dort greifbar werden zu lassen, wo diese nicht unbedingt als Verfahrensschritt betrachtet oder angekündigt sind (z.B. in der Literaturkritik oder in Kanonisierungsprozessen); eine weitere offenkundige Schwierigkeit ist die Historizität des Vergleichs und des Vergleichens.

Brandts Modell führt konsequent das Spezifische und eine potenziell verallgemeinerbare Anschauungsform in jeder ihrer Modellstufen zusammen, deshalb ließe sich diese schrittweise Aufschlüsselung der Integration auch für vertiefende Studien zur wissenschaftlichen Tradition des Vergleichens umsetzen. Die Metaphernforscherin

\footnotetext{
54 Willer, Stefan: »Die Allgemeinheit des Vergleichs. Ein komparatistisches Problem und seine Entstehung um 1800«. In: Michael Eggers (Hg.): Von Ähnlichkeiten und Unterschieden. Heidelberg 2011, S. 143-165 u. Ders.: »The problem of theorizing comparisons«. In: Neohelicon, Vol. 41 (2014), S. 371-380.
} 
Christine Brandt hat die Funktion von Metaphern als »Scharnier« betont; indem jene »konstitutiv für die Entstehung neuer Diskursformationen « seien, ${ }^{55}$ könnten sie zwischen literarischen und wissenschaftlichen Diskursen vermitteln. Metaphern bilden bekanntlich Schaltstellen zwischen Spezialdiskursen und werden als interdiskursive Elemente wirksam.

Der Darlegung der Vergleichsparameter einer jeden komparatistischen Untersuchung kommt in der akademischen Lehre eine große Bedeutung $\mathrm{zu}$, soll sie doch die Seriosität und Tragfähigkeit von Vergleichen untermauern. In Einführungswerken mag dieser Zwang zum Umgang mit Checklisten oder Suchrastern zum Standard gehören, ${ }^{56}$ in höheren komparatistischen Sphären scheint diese Vorgehensweise dann in der Regel weniger souverän. Die Voraussetzungen des Vergleichs werden wohl kaum bewusst verschleiert, aber sie können in den Hintergrund geraten oder sind womöglich im jeweiligen Begriffsrepertoire amalgamiert worden. Der vorliegende Beitrag will indessen herausstellen, dass die Mapping-Prozesse zwischen den Bedeutungsdomänen der comparata so genau wie möglich aufzuschlüsseln sind. Der Entwicklungsverlauf vom »base space« zur post-emergenten Bedeutung sollte nachgezeichnet werden, um den jeweiligen Ausgangspunkt der Hypothesenbildung sowie die pfadabhängigen Entscheidungen überhaupt der Reflexion zu erschließen. Nur so können vermeintlich selbstverständliche Vorannahmen des komparatistischen Vergleichs dingfest gemacht, immanente Widersprüchlichkeiten verfolgt sowie Alternativen vorstellbar und formulierbar werden.

Funding Open access funding provided by University of Vienna.

Open Access Dieser Artikel wird unter der Creative Commons Namensnennung 4.0 International Lizenz (http://creativecommons.org/licenses/by/4.0/deed.de) veröffentlicht, welche die Nutzung, Vervielfältigung, Bearbeitung, Verbreitung und Wiedergabe in jeglichem Medium und Format erlaubt, sofern Sie den/die ursprünglichen Autor(en) und die Quelle ordnungsgemäß nennen, einen Link zur Creative Commons Lizenz beifügen und angeben, ob Änderungen vorgenommen wurden.

\footnotetext{
55 Brandt, Christine: »Metapher«. In: Roland Borgards et al. (Hg.): Literatur und Wissen. Ein interdisziplinäres Handbuch. Stuttgart/Weimar 2013, S. 21-28, S. 25. Diese Forscherin weist jedoch einschränkend darauf hin, dass oftmals zweifelhafte dichotomische Strukturen in den methodischen Zugängen vorherrschten (vgl. ebd., S. 26). Außerdem entstünden die Bedeutungszuschüsse unkontrolliert - eine Position also, die Line Brandts Sichtweise widerspricht, da diese wie gezeigt das Mapping systematisch untersucht und einige exemplarische Strukturen ermittelt.

56 Vgl. Corbineau-Hoffmann, Angelika: Einführung in die Komparatistik. Berlin 2004, S. 113. Diese Autorin trifft mit einer hochschuldidaktischen Motivierung die Unterscheidung zwischen einer inhaltlichen und einer textorganisatorischen - kompositorischen - immanenten Interpretation, was auf eine strategisch ungünstige und anachronistische Trennung von Inhalt und Form hindeuten könnte. Für den inhaltlichen Vergleich wären dieser Autorin zufolge Thema, Motiv, Schauplätze, Figuren und eine sog. Konzeptebene heranzuziehen.
} 